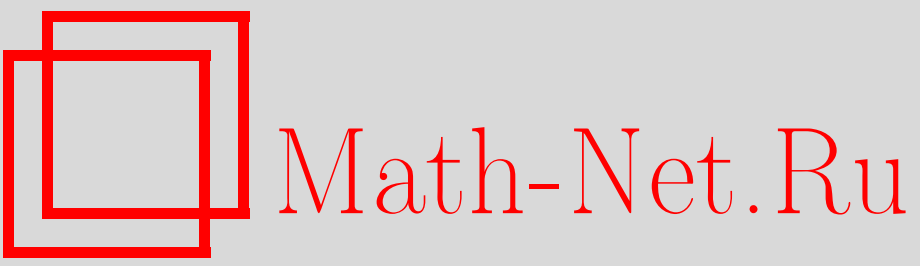

В. Н. Сачков, Цикловая структура элементов централизаторов подстановок симметрической группы, Матем. вопр. криптогр., 2010, том 1, выпуск 1, 85-100

DOI: https://doi.org/10.4213/mvk5

Использование Общероссийского математического портала Math-Net.Ru подразумевает, что вы прочитали и согласны с пользовательским соглашением

http://www . mathnet.ru/rus/agreement

Параметры загрузки:

IP : 54.164 .48 .24

26 апреля 2023 г., 15:48:42 
УДК 512.542

\title{
Цикловая структура элементов централизаторов подстановок симметрической группы
}

\author{
В. Н. Сачков \\ Академия криптографии Российской Федерации, Москва
}

Получено 22.IV.2010

Для числа циклов случайного элемента централизатора подстановки $A$ степени $n$ найдена производящая функция и описаны предельные распределения числа циклов при $n \rightarrow \infty$ и некоторых ограничениях на характер изменения подстановки $A$ с ростом $n$.

Ключевые слова: подстановки, производящие функции, централизатор, предельное распределение, циклы.

Cycle structure of the elements of centralizers of permutations from the symmetric group

\author{
V. N. Sachkov \\ Academy of Cryptography of Russian Federation, Moscow
}

\begin{abstract}
Let $\xi$ be the number of cycles of a random element of centralizer of a permutation from the symmetric group $S_{n}$ with given cycle structure. For some classes of permutations generating functions and limit distributions of $\xi$ as $n \rightarrow \infty$ are described.
\end{abstract}

Key words: permutation, centralizer, cycles, generating function, limit distribution.

Citation: Mathematical Aspects of Cryptography, 2010, vol. 1, no. 1, pp. 85-100 (Russian).

() 2010 В. Н. Сачков 


\section{Введение}

Централизатор элемента $A$ из симметрической группы $S_{n}$ является совокупностью всех решений $X$ уравнения

$$
A X=X A
$$

в группе $S_{n}$. Уравнения вида (1.1) представляют интерес в криптографии, например, при изучении дискретных функций, задаваемых биективными отображениями полей Галуа и имеющих трансляторы [1].

Для подстановки $A$, принадлежащей цикловому классу $\left[1^{\alpha_{1}} 2^{\alpha_{2}} \ldots n^{\alpha_{n}}\right]$, $\sum_{r=1}^{n} r \alpha_{r}=n$, в п. 1 устанавливается биекция множества решений уравнения (1.1) с полупрямым произведением $\Phi \lambda \mathrm{T}$, где Ф и Т - прямые произведения симметрических групп и групп циклических сдвигов соответственно. На этой основе формулируется алгоритм построения решений (1.1) с заданной цикловой структурой.

В п. 2 и п. 3 для случайной величины $\xi\left(1^{\alpha_{1}} 2^{\alpha_{2}} \ldots n^{\alpha_{n}}\right)$, равной числу циклов в случайно выбранном решении $X$ уравнения (1.1), когда $A$ принадлежит цикловому классу $\left[1^{\alpha_{1}} 2^{\alpha_{2}} \ldots n^{\alpha_{n}}\right], \sum_{r=1}^{n} r \alpha_{r}=n$, найдено выражение для производящей функции $f\left(t ; 1^{\alpha_{1}} 2^{\alpha_{2}} \ldots n^{\alpha_{n}}\right)$ этой случайной величины:

$$
\begin{aligned}
f\left(t ; 1^{\alpha_{1}} 2^{\alpha_{2}} \ldots n^{\alpha_{n}}\right) & =\prod_{r=1}^{n} \frac{\Gamma\left(\Psi(t ; r)+\alpha_{r}\right)}{\Gamma(\Psi(t ; r)) \Gamma\left(\alpha_{r}+1\right)}, \\
\Psi(t ; r) & =\frac{1}{r} \sum_{d \backslash r} \varphi\left(\frac{r}{d}\right) t^{d},
\end{aligned}
$$

где $\Gamma$ — гамма-функция, $\varphi$ - функция Эйлера.

Для случайной величины $\xi\left(1^{\alpha_{1}} 2^{\alpha_{2}} \ldots n^{\alpha_{n}}\right)$ получено представление в виде суммы независимых случайных величин:

$$
\xi\left(1^{\alpha_{1}} 2^{\alpha_{2}} \ldots n^{\alpha_{n}}\right)=\sum_{r=1}^{n} \xi\left(r^{\alpha_{r}}\right)
$$

где $\xi\left(r^{\alpha_{r}}\right)$ - число циклов случайного элемента централизатора регулярной подстановки, содержащей $\alpha_{r}$ циклов длины $r$.

В п. 4 показано, что при соответствующих нормировках случайной величины $\xi\left(r^{k}\right)$ в зависимости от поведения величины $\ln k / r$ при $n \rightarrow \infty$ распределения нормированных случайных величин сходятся либо к нормальному распределению, либо к распределению пуассоновского типа, либо 
к вырожденному распределению. Для сумм нормированных случайных величин $\xi\left(r_{1}^{\alpha_{\eta}}\right), \ldots, \xi\left(r_{\ell}^{\alpha_{r_{l}}}\right), \sum_{i=1}^{\ell} r_{i} \alpha_{r_{i}}=n$, указаны условия, при которых для $n \rightarrow \infty$ предельными являются либо нормальное распределение, либо распределение пуассоновского типа.

\section{1. Элементы централизатора с данной цикловой структурой}

Пусть $S_{n}$ - симметрическая группа, действующая на множестве $N_{n}=\{1,2, \ldots, n\}$, и подстановка $A \in S_{n}$ принадлежит цикловому классу $\left[1^{\alpha_{1}} 2^{\alpha_{2}} \ldots n^{\alpha_{n}}\right], \alpha_{1}+2 \alpha_{2}+\ldots+n \alpha_{n}=n$. Централизатор подстановки $A$ представляет собой совокупность всех решений $X$ уравнения

$$
A X=X A
$$

в группе $S_{n}$. Из равенства (1.1) следует, что трансформация некоторого цикла $A$ элементом централизатора $X$ совпадает с некоторым циклом той же длины подстановки $A$. В соответствии с этим существует такое разбиение

$$
N_{n}=N_{n}^{(1)} \cup N_{n}^{(2)} \cup \ldots \cup N_{n}^{(n)},
$$

что $A^{(r)}$ - регулярная подстановка, действующая на множестве $N_{n}^{(r)}$ и состоящая из всех циклов длины $r$ подстановки $A$, так что

$$
A=A^{(1)} A^{(2)} \ldots \ldots A^{(n)} .
$$

Любое решение $X$ уравнения (1.1) может быть представлено в виде

где $X^{(r)}$ - решение уравнения

$$
X=X^{(1)} X^{(2)} \ldots \ldots X^{(r)}
$$

$$
A^{(r)} X^{(r)}=X^{(r)} A^{(r)}, \quad r=1,2, \ldots \ldots, n .
$$

Для подстановки $A^{(r)}$ из циклового класса $\left[r^{k}\right]$ на элементах $N_{n}^{(r)}$ зададим отношение эквивалентности, считая эквивалентными все элементы, на которых действует один и тот же цикл длины $r$ подстановки $A^{(r)}$. Имеется $k$ классов эквивалентности, содержащих каждый $r$ элементов.

Подстановку степени $k$, действующую на соответствующем фактормножестве, будем называть фактор-подстановкой. Совокупность всех фактор-подстановок образует группу $\Phi_{k}$, изоморфную группе $S_{k}$. Циклы факторподстановки будем называть фактор-циклами. При фиксированных факторциклах множество решений уравнения (1.1) определяется всевозможными сдвигами один относительно другого циклов длины $r$, соответствие между которыми определяется фактор-подстановкой. Совокупность таких сдвигов образует группу $\mathrm{T}_{r}$, являющуюся прямым произведением циклических групп, отвечающих взаимным сдвигам циклов один относительно другого. 
Подстановке $A$, принадлежащей цикловому классу $\left[1^{\alpha_{1}} 2^{\alpha_{2}} \ldots n^{\alpha_{n}}\right]$, поставим в соответствие группы Ф и Т, представляющие собой прямые произведения вида:

$$
\begin{gathered}
\Phi=\Phi_{\alpha_{1}} \times \Phi_{\alpha_{2}} \times \ldots \times \Phi_{\alpha_{n}}, \\
\mathrm{~T}=\mathrm{T}_{1} \times \mathrm{T}_{2} \times \ldots \times \mathrm{T}_{n},
\end{gathered}
$$

где $\alpha_{1}+2 \alpha_{2}+\ldots+n \alpha_{n}=n$.

Тогда каждое решение $X=X_{\mathrm{g}}$ уравнения (1.1) определяется элементом $g$ группы $\mathrm{G}$, представляющей собой полупрямое произведение групп Ф и Т,

$$
G=\Phi \lambda T,
$$

причем $g=\varphi t \in G, \varphi \in \Phi, t \in T$.

Исходя из описания решений уравнения (1.1), можно привести алгоритм построения таких решений, которые имеют заданную цикловую структуру.

\section{Алгоритм.}

1. Для подстановки $A$ из циклового класса $\left[1^{\alpha_{1}} 2^{\alpha_{2}} \ldots n^{\alpha_{n}}\right]$ определяются блоки $N_{n}^{(1)}, N_{n}^{(2)}, \ldots, N_{n}^{(n)}$ разбиения (1.2).

2. Для каждого $r, 1 \leq r \leq n$, рассматривается уравнение

$$
A^{(r)} X^{(r)}=X^{(r)} A^{(r)},
$$

где $A^{(r)}$ - сужение $A$ на множество $N_{n}^{(r)}$, причем $A^{(r)}$ - регулярная подстановка, определенная на $N_{n}^{(r)}$ и имеющая $\alpha_{r}$ циклов длины $r$.

3. При заданном $r$ рассматривается множество всех факторподстановок степени $\alpha_{r}$. Для каждой фактор-подстановки $\sigma \in \Phi_{\alpha_{r}}$ находится ее разложение в произведение независимых циклов $\sigma=\sigma_{1} \sigma_{2} \ldots \sigma_{l}$. Осуществляются все взаимные сдвиги один относительно другого $r$-циклов подстановки $A$, на которых действует цикл $\sigma_{j}, 1 \leq j \leq l$, фактор-подстановки $\sigma$. В результате получается множество решений $X^{(r)}$ уравнения (1.9), соответствующих фактор-подстановке $\sigma \in \Phi_{\alpha_{r}}, 1 \leq r \leq n$.

4. Операции проводятся для всех $r=1,2, \ldots, n$. В результате находится множество всех решений уравнения (1.1), каждое из которых имеет представление в виде произведения

$$
X=X^{(1)} X^{(2)} \ldots X^{(n)} .
$$

Отметим, что указанный алгоритм построения всех решений уравнения (1.1) фактически состоит в переборе всех элементов группы $G$, причем каждому элементу $g \in G$ отвечает одно и только одно решение. Алгоритм по- 
зволяет без перебора всех решений отдельно находить решения с данной цикловой структурой. Для этого на определенных шагах алгоритма на параметры накладываются соответствующие ограничения, определяемые заданной цикловой структурой решения.

\section{2. Число циклов решения для регулярной подстановки $A$}

Рассмотрим решения $X$ уравнения (1.1) для случая, когда подстановка $A$ степени $k r$ принадлежит цикловому классу $\left[r^{k}\right]$.

Лемма 1. Пусть А принадлежсит иикловому классу $\left[r^{k}\right]$, фиксированная фактор-подстановка полночикловая, $u v_{1}, v_{2}, \ldots, v_{k}-$ величины взаимных ииклических сдвигов $k$ циклов, порядок следования которых определяется фактор-подстановкой. Тогда решение уравнения $A X=X A$ является регулярной подстановкой, имеющей $d$ ииклов длины $\mathrm{kr} / \mathrm{d}$, где $d=\left(v_{1}+\ldots+v_{k}, r\right)$.

Действительно, если решение имеет цикл длины $l$, то из способа построения решений следует, что $\ell=k v$, где $v-$ наименьшее положительное решение уравнения

$$
v\left(v_{1}+\ldots+v_{k}\right) \equiv 0(\bmod r), 0 \leq v_{i} \leq r-1,1 \leq i \leq k .
$$

Если $d=\left(v_{1}+\ldots+v_{k}, r\right)$, то $v=r / d$ и, стало быть, $\ell=k r / d$, и число циклов равно $d$.

Для случая, когда $A$ принадлежит цикловому классу $\left[r^{k}\right]$, на множестве решений уравнения (1.1), для которых фактор-подстановка зафиксирована и является полноцикловой, зададим равномерное вероятностное распределение и рассмотрим случайную величину $\xi_{r}$, равную числу циклов в случайно выбранном решении.

В соответствии с леммой 1 имеем:

$$
\mathbf{P}\left(\xi_{r}=d\right)=\frac{1}{r^{k}} \sum_{\substack{j=0 \\(j, r)=d}} \sum_{\substack{v_{1}+\ldots+v_{k}=j(\bmod r) \\ 0 \leq v_{i} \leq r-1,1 \leq i \leq k}} 1
$$

и

$$
\sum_{\substack{v_{1}+\ldots+v_{k}=j(\bmod r) \\ 0 \leq v_{i} \leq r-1,1 \leq i \leq k}} 1=\sum_{l=0}^{k-1} \sum_{\substack{v_{1}+\ldots+v_{k}=j+l k \\ 0 \leq v_{i} \leq r-1,1 \leq i \leq k}} 1 .
$$

Так как

$$
\sum_{\substack{v_{1}+\ldots+v_{k}=j+l r \\ 0 \leq v_{i} \leq r-1,1 \leq i \leq k}} 1=\operatorname{coef}_{x^{j+l}}\left(1+x+x^{2}+\ldots+x^{r-1}\right)^{k}
$$


то, используя первообразный корень $\alpha$ степени $n$ из единицы, получаем, что

$$
\sum_{v_{1}+\ldots+v_{k} \equiv j(\bmod r)} 1=\frac{1}{r} \sum_{s=1}^{r} \alpha^{-j s}\left(1+\alpha^{s}+\alpha^{2 s}+\ldots+\alpha^{(r-1) s}\right)^{k}=r^{k-1} .
$$

Из соотношений (2.2) и (2.3) получаем окончательное выражение для распределения $\xi_{r}$ :

$$
\mathbf{P}\left(\xi_{r}=d\right)=\frac{1}{r} \varphi\left(\frac{r}{d}\right), \quad d \backslash r,
$$

где $\varphi(x)$ - функция Эйлера.

Производящая функция для $\xi_{r}$ имеет вид

$$
\Psi(t ; r)=\frac{1}{r} \sum_{d \backslash r} \varphi\left(\frac{r}{d}\right) t^{d} .
$$

Отсюда следует выражение для характеристической функции случайной величины $\xi_{r}$ :

$$
\Psi\left(e^{i t} ; r\right)=\frac{1}{r} \sum_{d \backslash r} \varphi\left(\frac{r}{d}\right) e^{i d t} .
$$

Эта функция дифференцируема в окрестности точки $t=0$ и, следовательно, для моментов $\mathbf{M} \xi_{r}^{s}$ случайной величины $\xi_{r}$ имеем:

$$
\mathbf{M} \xi_{r}^{s}=\left.\frac{1}{i^{s}} \frac{d^{s}}{d t^{s}} \Psi\left(e^{i t}, r\right)\right|_{t=0}=r^{s-1} \sum_{d \backslash r} \frac{1}{d^{s}} \varphi(d), s=1,2, \ldots
$$

где $\frac{d^{s}}{d t^{s}} \Psi\left(e^{i t} ; r\right)-s$-я производная функции $\Psi\left(e^{i t} ; r\right)$.

Пусть $r$ имеет каноническое разложение

$$
r=r_{1}^{\gamma_{1}} r_{2}^{\gamma_{2}} \ldots r_{v}^{\gamma_{v}}
$$

Тогда

$$
\varphi(r)=r\left(1-\frac{1}{r_{1}}\right) \ldots\left(1-\frac{1}{r_{v}}\right),
$$

и с использованием формулы (2.7) моменты $\mathbf{M} \xi_{r}$ могут быть представлены в следующем виде:

$$
\mathbf{M} \xi_{r}^{s}=\theta_{s}(r) r^{s-1}, s=1,2, \ldots
$$

где

$$
\theta_{1}(r)=\prod_{i=1}^{v}\left(1+\gamma_{i}\left(1-\frac{1}{r_{i}}\right)\right)
$$




$$
\theta_{s}(r)=\prod_{i=1}^{v}\left(1+\frac{1}{r_{i}^{s-1}}\left(1-\frac{1}{r_{i}}\right) \frac{1-1 / r_{i}^{(s-1) \gamma_{i}}}{1-1 / r_{i}^{(s-1)}}\right), s=2,3, \ldots
$$

Отметим, что в соответствии с (2.8) $\tau(r)$ - число делителей $r$ определяется формулой

$$
\tau(r)=\prod_{i=1}^{v}\left(1+\gamma_{i}\right) .
$$

Для последующих асимптотических оценок потребуются три леммы.

Лемма 2. Если при $r \rightarrow \infty$ для канонических разложений выполнено условие

$$
\sum_{i=1}^{v} \frac{1}{r_{i}} \rightarrow 0
$$

то имеет место сходимость

$$
\frac{\mathbf{M} \xi_{r}}{\tau(r)} \rightarrow 1
$$

Действительно, из формул (2.10) и (2.12) имеем:

$$
1-\sum_{i=1}^{v} \frac{1}{r_{i}} \leq \frac{\mathbf{M} \xi_{r}}{\tau(r)} \leq 1
$$

из этих неравенств следует лемма 2.

Лемма 3. При $r \rightarrow \infty$ справедливо соотночение

$$
\frac{\mathrm{M} \xi_{r}}{r} \rightarrow 0 .
$$

Соотношение (2.15) следует из неравенств

$$
\prod_{i=1}^{v}\left(\frac{1+\gamma_{i}}{r_{i}^{\gamma_{i}}}\right)\left(1-\sum_{i=1}^{v} \frac{1}{r_{i}}\right) \leq \frac{\mathbf{M} \xi_{r}}{r} \leq \prod_{i=1}^{v}\left(\frac{1+\gamma_{i}}{r_{i}^{\gamma_{i}}}\right),
$$

вытекающих из формул (2.10) и (2.12) и соотношения

$$
\prod_{i=1}^{v}\left(\frac{1+\gamma_{i}}{r_{i}^{\gamma_{i}}}\right) \rightarrow 0 \text {, при } r \rightarrow \infty \text {. }
$$

Для оценки моментов $\mathbf{M} \xi_{r}^{s}$ при $r \rightarrow \infty$ будем использовать следующую лемму. 
Лемма 4. Если при $r \rightarrow \infty$ выполнено условие (2.13), то имеют место асимптотические оценки

$$
\theta_{s}(r)=1+O\left(\sum_{i=1}^{v} \frac{1}{r_{i}^{s-1}}\right), s \geq 2,
$$

u, следовательно, существуют предель

$$
\theta_{s}(r) \rightarrow 1, \quad s=2,3, \ldots .
$$

Лемма 4 следует из формул (2.11) и вытекающих из них неравенств

$$
1-\sum_{i=1}^{v} \frac{1}{r_{i}^{s}+r_{i}} \leq \frac{\theta_{s}(r)}{\prod_{i=1}^{v}\left(1+\frac{1}{r_{i}^{s-1}}\right)} \leq 1, \quad s \geq 2 .
$$

Очевидно, что из условия (2.13) следует, что

$$
\sum_{i=1}^{v} \frac{1}{r_{i}^{s-1}} \rightarrow 0, s=2,3, \ldots
$$

Из формул (2.9), (2.13) и леммы 4 следует, что при $r \rightarrow \infty$

$$
\frac{\mathbf{M} \xi_{r}^{s}}{r^{s-1}} \rightarrow 1, s=2,3, \ldots
$$

Рассмотрим теперь характеристическую функцию случайной величины $\xi_{r} / r$, которая в силу условий (2.20) для достаточно больших $r$ в некоторой окрестности точки $t=0$ может быть представлена в виде сходящегося ряда

$$
\Psi\left(e^{i t} ; r\right)=1+\sum_{s=1}^{\infty} \frac{\mathbf{M} \xi_{r}^{s}}{r^{s}} \frac{(i t)^{s}}{s !} .
$$

Из равенства (2.21) имеем:

$$
\Psi\left(e^{i t / r} ; r\right)=1+\frac{\theta_{1}(r)}{r} \frac{i t}{1 !}+\frac{1}{r} \sum_{s=2}^{\infty} \theta_{s}(r) \frac{(i t)^{s}}{s !} .
$$

Из лемм 3 и 4 и формулы (2.22) следует, что для любого $t$ при условии (2.13) и $r \rightarrow \infty$

$$
\Psi\left(e^{i t / r} ; r\right) \rightarrow 1
$$

\section{3. Производящие функции и моменты для числа циклов в случайно выбранном решении}

Обозначим через $\xi\left(r^{k}\right)$ случайную величину, равную числу циклов в случайно выбранном решении уравнения (1.1), когда подстановка $A$ при- 
надлежит цикловому классу $\left[r^{k}\right]$. Рассмотрим производящую функцию величины $\xi\left(r^{k}\right)$ :

$$
f\left(t ; r^{k}\right)=\sum_{s=1}^{r k} \mathbf{P}\left(\xi\left(r^{k}\right)=s\right) t^{s} .
$$

Лемма 5. Производящая функиия $f\left(t ; r^{k}\right)$ имеет следующее выражение:

$$
f\left(t ; r^{k}\right)=\frac{\Gamma(\Psi(t ; r)+k)}{\Gamma(\Psi(t ; r)) \Gamma(k+1)},
$$

где $\Gamma(x)$ - гамма-функция.

Действительно, если $\xi\left(r^{k} ; v_{1}, \ldots, v_{k}\right)-$ случайная величина, равная числу циклов в случайно выбранном решении уравнения (1.1), когда $A$ принадлежит цикловому классу $\left[r^{k}\right]$, а фактор-подстановка выбрана из циклового класса $\left[1^{v_{1}} 2^{v_{2}} \ldots k^{v_{k}}\right], v_{1}+2 v_{2}+\ldots+k v_{k}=k$, то для производящей функции этой случайной величины имеем:

$$
f\left(t ; r^{k}, v_{1}, \ldots, v_{k}\right)=\sum_{s=1}^{r k} \mathbf{P}\left(\xi\left(r^{k}, v_{1}, \ldots, v_{k}\right)=s\right) t^{k s}=[\Psi(t ; r)]^{v_{1}+v_{2}+\ldots+v_{k}},
$$

где $\Psi(t ; r)$ задана формулой (2.5).

Производя усреднение по всем цикловым классам, которым может принадлежать фактор-подстановка, получаем:

$$
f\left(t ; r^{k}\right)=\sum_{\sum_{i=1}^{k} i v_{i}=k} \frac{1}{1^{v_{1}} 2^{v_{2}} \ldots k^{v_{k}} v_{1} ! v_{2} ! \ldots v_{k} !}[\Psi(t ; r)]^{v_{1}+\ldots+v_{k}} .
$$

Отсюда следует, что

$$
f\left(t ; r^{k}\right)=\frac{1}{k !} C_{k}(\Psi(t ; r), \ldots, \Psi(t ; r)),
$$

где $\frac{1}{k !} C_{k}\left(x_{1}, x_{2}, \ldots, x_{k}\right)$ - циклический индекс симметрической группы $S_{k}$ [2]. Следовательно,

$$
f\left(t ; r^{k}\right)=\frac{1}{k !} \Psi(t ; r)(\Psi(t ; r)+1) \ldots(\Psi(t ; r)+k-1) .
$$

Из формулы (3.3) следует формула (3.2).

Обозначим через $\xi\left(1^{\alpha_{1}} 2^{\alpha_{2}} \ldots n^{\alpha_{n}}\right)$ число циклов в случайно выбранном решении уравнения (1.1), когда $A$ принадлежит цикловому классу $\left[1^{\alpha_{1}} 2^{\alpha_{2}} \ldots n^{\alpha_{n}}\right]$, 
$\alpha_{1}+2 \alpha_{2}+\ldots+n \alpha_{n}=n$. Тогда в соответствии с алгоритмом построения решений уравнения (1.1) $\xi\left(1^{\alpha_{1}} 2^{\alpha_{2}} \ldots n^{\alpha_{n}}\right)$ представляет собой сумму независимых случайных величин:

$$
\xi\left(1^{\alpha_{1}} 2^{\alpha_{2}} \ldots n^{\alpha_{n}}\right)=\sum_{r=1}^{n} \xi\left(r^{\alpha_{r}}\right),
$$

и, следовательно, в соответствии с формулой (3.2) для производящей функции

$$
f\left(t ; 1^{\alpha_{1}} 2^{\alpha_{2}} \ldots n^{\alpha_{n}}\right)=\sum_{s=1}^{n} \mathbf{P}\left(\xi\left(1^{\alpha_{1}} 2^{\alpha_{2}} \ldots n^{\alpha_{n}}\right)=s\right) t^{s}
$$

имеет место формула

$$
f\left(t ; 1^{\alpha_{1}} 2^{\alpha_{2}} \ldots n^{\alpha_{n}}\right)=\prod_{r=1}^{n} \frac{\Gamma\left(\Psi(t ; r)+\alpha_{r}\right)}{\Gamma(\Psi(t ; r)) \Gamma\left(\alpha_{r}+1\right)}, \sum_{r=1}^{n} r \alpha_{r}=n .
$$

Путем отыскания первых двух производных функций $f\left(t ; r^{k}\right)$ с использованием формулы (3.3) находим выражения для среднего и дисперсии $\xi\left(r^{k}\right)$ :

$$
\begin{gathered}
m\left(r^{k}\right)=\mathbf{M} \xi\left(r^{k}\right)=\sum_{d \backslash r} \frac{\varphi(d)}{d} \sum_{j=1}^{k} \frac{1}{j} \\
\sigma^{2}\left(r^{k}\right)=\mathbf{D} \xi\left(r^{k}\right)=r \sum_{d \backslash r} \frac{\varphi(d)}{d} \sum_{j=1}^{k} \frac{1}{j}-\left[\sum_{d \backslash r} \frac{\varphi(d)}{d}\right]^{2} \sum_{j=1}^{k} \frac{1}{j^{2}} .
\end{gathered}
$$

Если $r$ имеет каноническое разложение вида (2.8), то из формул (3.6) и (3.7) с учетом равенств (2.10) и (2.11) следует, что

$$
\begin{gathered}
m\left(r^{k}\right)=\theta_{1}(r) \sum_{j=1}^{k} \frac{1}{j}, \\
\sigma^{2}\left(r^{k}\right)=r \theta_{2}(r) \sum_{j=1}^{k} \frac{1}{j}-\theta_{1}^{2}(r) \sum_{j=1}^{k} \frac{1}{j^{2}} .
\end{gathered}
$$

При $k \rightarrow \infty$ из формул (3.8) и (3.9) находим, что

$$
\begin{gathered}
m\left(r^{k}\right)=\theta_{1}(r) \ln k\left(1+O\left(\frac{1}{\ln k}\right)\right), \\
\sigma^{2}\left(r^{k}\right)=r \theta_{2}(r) \ln k\left(1+O\left(\frac{r}{\ln k}\right)\right) .
\end{gathered}
$$


В соответствии с равенствами (3.4), (3.8) и (3.9) среднее и дисперсия $\xi\left(1^{\alpha_{1}} 2^{\alpha_{2}} \ldots n^{\alpha_{n}}\right)$ имеют вид:

$$
\begin{gathered}
m\left(1^{\alpha_{1}} 2^{\alpha_{2}} \ldots n^{\alpha_{n}}\right)=\mathbf{M} \xi\left(1^{\alpha_{1}} 2^{\alpha_{2}} \ldots n^{\alpha_{n}}\right)=\sum_{r=1}^{n} \theta_{1}(r) \sum_{j=1}^{\alpha_{r}} \frac{1}{j}, \\
\sigma^{2}\left(1^{\alpha_{1}} 2^{\alpha_{2}} \ldots n^{\alpha_{n}}\right)=\mathbf{D}\left(\xi\left(1^{\alpha_{1}} 2^{\alpha_{2}} \ldots n^{\alpha_{n}}\right)\right)=\sum_{r=1}^{n}\left[r \theta_{2}(r) \sum_{j=1}^{\alpha_{r}} \frac{1}{j}-\theta_{2}^{2}(r) \sum_{j=1}^{\alpha_{r}} \frac{1}{j^{2}}\right],
\end{gathered}
$$

где $\sum_{r=1}^{n} r \alpha_{r}=n$.

\section{4. Предельная теорема}

Предельное поведение при $k \rightarrow \infty$ случайной величины $\xi\left(r^{k}\right)$ описывается следующей теоремой.

Теорема. При $k \rightarrow \infty$ в зависимости от скорости роста r имеются три случая:

$1^{0}$. Если $\frac{\ln k}{r} \rightarrow \infty$, то случайная величина $\xi^{\prime}=\left(\xi\left(r^{k}\right)-m\left(r^{k}\right)\right) \sigma^{-1}\left(r^{k}\right)$ в пределе имеет нормальное распределение с параметрами $(0,1)$.

$2^{0}$. Если $\frac{\ln k}{r} \rightarrow \lambda>0$ и каноническое разложение $r$ вида (2.8) удовлетворяет условию (2.13), то функиия распределения случайной величины $\xi^{\prime \prime}=\left(\xi\left(r^{k}\right)-\left(\theta_{1}(r)-1\right) \ln k\right) r^{-1}$ слабо сходится к функиии распределения пуассоновского типа

$$
F(x ; \lambda)=e^{-\lambda} \sum_{j=0}^{[x]} \frac{\lambda^{j}}{j !},
$$

где $[x]$ - иелая часть от х. В точках разрыва функиии $F(x ; \lambda)$ сходимость в общем случае не имеет места.

$3^{0}$. Если $\frac{\ln k}{r} \rightarrow 0$ и выполнены соотномения (2.8) и (2.13), то предельное распределение случайной величины $\xi\left(r^{k}\right) / r$ вырожденное, а именно, для любого $\varepsilon>0$

$$
\mathbf{P}\left(\left|\frac{\xi\left(r^{k}\right)}{r}-\frac{\left(\theta_{1}(r)-1\right) \ln k}{r}\right|<\varepsilon\right) \rightarrow 1 .
$$




\section{Доказательство}

$1^{0}$. Пусть $\ln k / r \rightarrow \infty$. Положим $m=m\left(r^{k}\right)$ и $\sigma^{2}=\sigma^{2}\left(r^{k}\right)$. Из формул (2.9), (3.10) и (3.11) следует, что

$$
\begin{gathered}
\frac{m}{\sigma}=\theta_{1}(r) \sqrt{\frac{\ln k}{r \theta_{2}(r)}}\left(1+O\left(\frac{r}{\ln k}\right)\right), \\
\frac{\mathbf{M} \xi_{r}^{2}}{\sigma^{2}} \ln k=1+O\left(\frac{r}{\ln k}\right) .
\end{gathered}
$$

Кроме того,

$$
\frac{\mathbf{M} \xi_{r}^{3}}{\sigma^{3}} \ln k=O\left(\sqrt{\frac{r}{\ln k}}\right) .
$$

У случайной величины $\xi_{r} / \sigma$ существуют первые три момента, и для ее характеристической функции в окрестности нуля имеет место представление

$$
\Psi\left(e^{i t / \sigma} ; r\right)=1+\mathbf{M} \xi_{r} \frac{i t}{\sigma}-\frac{t^{2}}{2 \sigma^{2}} \mathbf{M} \xi_{r}^{2}+R(t ; r)
$$

где

$$
|R(t ; r)| \leq \frac{|t|^{3}}{6 \sigma^{3}} \mathbf{M} \xi_{r}^{3} .
$$

С использованием формулы (3.2) и формулы Стирлинга для Г-функции найдем асимптотическое представление для логарифма характеристической функции $\chi\left(t ; r^{k}\right)$ случайной величины $\left(\xi\left(r^{k}\right)-m\right) \sigma^{-1}$. Имеем:

$$
\ln \chi\left(t ; r^{k}\right)=-\frac{i m t}{\sigma}+\left(\Psi\left(e^{i t / \sigma} ; r\right)-1\right) \ln k-\ln \Gamma\left(\Psi\left(e^{i t / \sigma} ; r\right)\right)+O\left(\frac{r \ln k}{k}\right) .
$$

Из соотношения (4.5) с учетом формул (4.2), (4.3) и (4.4) следует, что

$$
\Psi\left(e^{i t / \sigma} ; r\right) \rightarrow 1,
$$

а из равенства (4.6) получаем, что при любом $t$

$$
\ln \chi\left(t ; r^{k}\right)=-\frac{t^{2}}{2}+O\left(\sqrt{\frac{r}{\ln k}}\right) .
$$

Отсюда следует, что при любом $t$

$$
\chi\left(t ; r^{k}\right) \rightarrow e^{-t^{2} / 2}
$$


$2^{0}$. Пусть $\frac{\ln k}{r} \rightarrow \lambda>0$ и выполнено условие (2.13). Тогда, используя формулу (3.2) и применяя формулу Стирлинга для оценки Г-функции при $k \rightarrow \infty$, получаем асимптотическое представление для логарифма характеристической функции $\tilde{\chi}\left(t ; r^{k}\right)$ случайной величины $\xi^{\prime \prime}$ :

$$
\begin{gathered}
\ln \tilde{\chi}\left(t ; r^{k}\right)=-\frac{i t\left(\theta_{1}(r)-1\right) \ln k}{r}+ \\
+\left(\Psi\left(e^{i t / r} ; r\right)-1\right) \ln k-\ln \Gamma\left(\Psi\left(e^{i t / r} ; r\right)\right)+O\left(\frac{\ln k}{k}\right) .
\end{gathered}
$$

Из соотношения (2.23) следует, что при $r \rightarrow \infty$

$$
\ln \Gamma\left(\Psi\left(e^{i t / r} ; r\right)\right) \rightarrow 0 \text {. }
$$

Используя равенство (2.22), из соотношения (4.8) получаем, что

$$
\ln \tilde{\chi}\left(t ; r^{k}\right)=\frac{\ln k}{r} i t+\frac{\ln k}{r} \sum_{s=2}^{\infty} \theta_{s}(r) \frac{(i t)^{s}}{s !}+O\left(\frac{\ln k}{k}\right) .
$$

При выполнении условия (2.13) для $s=2,3, \ldots$ имеют место соотношения (2.17), из которых вытекает существование пределов (2.18). Это означает, что при достаточно больших $r$ величины $\theta_{s}(r)$ ограничены и, следовательно, ряд в правой части равенства (4.10) сходится равномерно в некоторой ограниченной окрестности точки $t=0$. Поэтому при выполнении условия $2^{0}$ теоремы при $k, r \rightarrow \infty$ получаем, что

$$
\ln \tilde{\chi}\left(t ; r^{k}\right)=\lambda\left(e^{i t}-1\right)\left(1+O\left(\sum_{i=1}^{v} \frac{1}{r_{i}}\right)\right)+O\left(\frac{\ln k}{k}\right) .
$$

Переходя к пределу при $k, r \rightarrow \infty$, с учетом условий $2^{0}$ получаем, что для любого $t$

$$
\tilde{\chi}\left(t ; r^{k}\right) \rightarrow e^{\lambda\left(e^{i t}-1\right)} .
$$

$3^{0}$. При $\frac{\ln k}{r} \rightarrow 0$ и $k \rightarrow \infty$ соотношение (4.1) следует из существования при этих условиях предела $\tilde{\chi}\left(t ; r^{k}\right) \rightarrow 1$. Теорема доказана.

Пусть теперь $A$ принадлежит цикловому классу $\left[r_{1}^{\alpha_{\eta}} \ldots r_{l}^{\alpha_{\eta}}\right]$, $r_{1} \alpha_{r_{1}}+\ldots+r_{l} \alpha_{r_{l}}=n$, и $\xi\left(r_{1}^{\alpha_{r_{1}}} \ldots r_{l}^{\alpha_{\eta_{1}}}\right)-$ число циклов в случайном выбранном решении $X$ уравнения (1.1). Если числа $r_{1}, \ldots, r_{l}$ имеют канонические разложения

$$
r_{j}=r_{1 j}{ }^{\gamma_{1 j}} \ldots r_{v_{j}, j}^{\gamma_{j, j}}, j=1,2, \ldots, l,
$$


то получаем следующие формулы для средних и дисперсий:

$$
\begin{gathered}
m_{j}=\mathbf{M} \xi\left(r_{j}^{\alpha_{r_{j}}}\right)=\prod_{i=1}^{v_{j}}\left[1+\gamma_{i j}\left(1-\frac{1}{r_{i j}}\right)\right] \ln \alpha_{r_{j}}, \\
\sigma_{j}^{2}=\mathbf{D} \xi\left(r_{j}^{\alpha_{r_{j}}}\right)=r_{j} \prod_{i=1}^{v_{j}}\left[1+\frac{1}{r_{i j}}\left(1-\frac{1}{r_{i j}^{\gamma_{j j}}}\right)\right] \ln \alpha_{r_{j}}, \\
j=1,2, \ldots, l .
\end{gathered}
$$

Из приведенной выше теоремы получаем два следствия.

Следствие 1. При $n \rightarrow \infty$ и выполнении условий

$$
\frac{\ln \alpha_{r_{j}}}{r_{j}} \rightarrow \infty, j=1,2, \ldots, l,
$$

для предельного распределения случайной величины

$$
\eta\left(r_{1}{ }^{\alpha_{r_{1}}} \ldots r_{l}^{\alpha_{r_{l}}}\right)=\sum_{j=1}^{l} \frac{\xi\left(r_{j}^{\alpha_{r_{j}}}\right)-m_{j}}{\sigma_{j}}
$$

могут быть два случая:

a) если $0<l \leq c<\infty$, то случайная величина $\eta\left(r_{1}^{\alpha_{r_{1}}}, \ldots, r_{l}^{\alpha_{r_{l}}}\right)$ асимптотически нормальна с параметрами $(0, l)$;

б) если $l \rightarrow \infty$ и выполнено условие

$$
\sum_{j=1}^{l} \sqrt{\frac{r_{j}}{\ln \alpha_{r_{j}}}} \rightarrow 0,
$$

то случайная величина $\eta\left(r_{1}^{\alpha_{r_{1}}}, \ldots, r_{l}^{\alpha_{r_{l}}}\right) / \sqrt{l}$ имеет в пределе нормальное распределение с параметрами $(0,1)$.

Условие а) следствия 1 следует из условия $1^{0}$ теоремы 1 , так как сумма независимых нормально распределенных случайных величин имеет нормальное распределение.

При выполнении условий б) для логарифма характеристической функции величины $\left(\xi\left(r_{j}^{\alpha_{r_{j}}}\right)-m_{j}\right) \sigma_{j}^{-1}$ из соотношения (4.7) следует выражение

$$
\ln \chi\left(t ; r_{j}^{\alpha_{r_{j}}}\right)=-\frac{t^{2}}{2}+O\left(\sqrt{\frac{r_{j}}{\ln \alpha_{r_{j}}}}\right) .
$$


Поэтому для логарифма характеристической функции величины $\eta\left(r_{1}^{\alpha_{\eta}}, \ldots, r_{l}^{\alpha_{\eta}}\right) / \sqrt{l}$ имеем следующее представление:

$$
\ln \chi\left(t ; r_{j}^{\alpha_{r_{j}}}, \ldots, r_{l}^{\alpha_{r_{l}}}\right)=-\frac{t^{2}}{2}+O\left(\sum_{j=1}^{l} \sqrt{\frac{r_{j}}{\ln \alpha_{r_{j}}}}\right),
$$

из которого в силу условия (4.16) находим, что

$$
\ln \chi\left(t ; r_{j}^{\alpha_{r_{j}}}, \ldots, r_{l}^{\alpha_{\eta}}\right) \rightarrow e^{-t^{2} / 2}
$$

Следствие 2. Если при $n \rightarrow \infty$ выполнены условия

$$
\frac{\ln \alpha_{r_{j}}}{r_{j}} \rightarrow \lambda_{j}>0, j=1,2, \ldots, l,
$$

и канонические разложения $r_{1}, r_{2}, \ldots, r_{l}$ удовлетворяют соотношениям

$$
\sum_{j=1}^{l} \sum_{i=1}^{\lambda_{j}} \frac{1}{r_{i j}} \rightarrow 0, \sum_{j=1}^{l} \frac{\ln \alpha_{r_{j}}}{\alpha_{r_{j}}} \rightarrow 0,
$$

то для предельных распределений случайной величины

$$
\zeta\left(r_{1}^{\alpha_{\eta}}, \ldots, r_{l}^{\alpha_{\eta}}\right)=\sum_{j=1}^{l} \frac{\xi\left(r_{j}^{\alpha_{r_{j}}}\right)-\left(\theta_{1}\left(r_{j}\right)-1\right) \ln \alpha_{r_{j}}}{r_{j}}
$$

имеют место два случая:

а) если $\lambda=\lambda_{1}+\ldots+\lambda_{l}<\infty, 0<l \leq c<\infty$, то функция распределения случайной величины $\zeta\left(r_{1}^{\alpha_{r_{1}}}, \ldots, r_{l}^{\alpha_{r_{l}}}\right)$ слабо сходится $\kappa$ функиии распределения пуассоновского типа с параметром $\lambda_{1}+\ldots+\lambda_{l}$;

б) если $\lambda=\lambda_{1}+\ldots+\lambda_{1} \rightarrow \infty, \quad l \rightarrow \infty, \quad$ то случайная величина $\left(\zeta\left(r_{1}^{\alpha_{r l n}} \ldots r_{j}^{\alpha_{r j}}\right)-\lambda\right) / \sqrt{\lambda}$ асимптотически нормальна с параметрами $(0,1)$.

Случай а) следствия 2 следует из условия $2^{0}$ теоремы 1 , так как распределение суммы независимых случайных величин, распределение каждой из которых сходится к распределению пуассоновского типа, слабо сходится к свертке указанных распределений. При выполнении условий б) в соответ- 
ствии с равенством (4.11) для логарифма характеристической функции величины $\left(\zeta\left(r_{1}^{\alpha_{r_{1}}}, \ldots, r_{l}^{\alpha_{r_{l}}}\right)-\lambda\right) / \sqrt{\lambda}$ имеем представление

$$
\begin{gathered}
\ln \tilde{\chi}\left(t ; r_{1}^{\alpha_{r_{1}}}, \ldots, r_{l}^{\alpha_{r_{i}}}\right)= \\
-i t \sqrt{\lambda} \sum_{j=1}^{l} \ln \tilde{\chi}\left(\frac{t}{\sqrt{\lambda}} ; r_{j}^{\alpha_{r_{j}}}\right)=-\frac{t^{2}}{2}+O\left(\frac{1}{\sqrt{\lambda}}\right)+O\left(\sum_{j=1}^{l} \sum_{i=1}^{\lambda_{j}} \frac{1}{r_{i j}}\right)+O\left(\sum_{j=1}^{l} \frac{\ln \alpha_{r_{j}}}{\alpha_{r_{j}}}\right),
\end{gathered}
$$

из которого следует, что для любого $t$

$$
\tilde{\chi}\left(t ; r_{1}^{\alpha_{r_{1}}}, \ldots, r_{l}^{\alpha_{r_{l}}}\right) \rightarrow e^{-t^{2} / 2}
$$

\section{Список литературы}

1. Сачков В. Н. Трансляторы и трансляции дискретных функций. - В сб.: Труды по дискретной математике. Т. 9. - М.: Гелиос, 2006, с. 253-268.

2. Сачков В.Н. Комбинаторные методы дискретной математики. - М.: Наука, 1977. 